\title{
DARK MATTER HALO MERGERS: DEPENDENCE ON ENVIRONMENT
}

\author{
J. A. Hester ${ }^{1}$ AND A. TASITSIOMI ${ }^{2,3}$ \\ ${ }^{1}$ California Institute of Technology, Pasadena, CA 91125, USA; jhester@ srl.caltech.edu \\ ${ }^{2}$ Department of Astrophysical Sciences, Peyton Hall, Princeton University, Princeton, NJ 08540, USA \\ Received 2008 September 16; accepted 2010 April 4; published 2010 April 28
}

\begin{abstract}
This paper presents a study of the specific major merger rate as a function of group membership, local environment, and redshift in a very large, $500 \mathrm{~h}^{-1} \mathrm{Mpc}$, cosmological $\mathrm{N}$-body simulation, the Millennium Simulation. The goal is to provide environmental diagnostics of major merger populations in order to test simulations against observations and provide further constraints on major merger driven galaxy evolution scenarios. A halo sample is defined using the maximum circular velocity, which is both well defined for subhalos and closely correlated with galaxy luminosity. Subhalos, including the precursors of major mergers, are severely tidally stripped. Major mergers between subhalos are therefore rare compared to mergers between subhalos and their host halos. Tidal stripping also suppresses dynamical friction, resulting in long major merger timescales when the more massive merger progenitor does not host other subhalos. When other subhalos are present, however, major merger timescales are several times shorter. This enhancement may be due to inelastic unbound collisions between subhalos, which deplete their orbital angular momentum and lead to faster orbital decay. Following these results, we predict that major mergers in group environments are dominated by mergers involving the central galaxy, that the specific major merger rate is suppressed in groups when all group members are considered together, and that the frequency of fainter companions is enhanced for major mergers and their remnants. We also measure an "assembly bias" in the specific major merger rate in that major mergers of galaxy-like halos are slightly suppressed in overdense environments while major mergers of group-like halos are slightly enhanced. A dynamical explanation for this trend is advanced which calls on both tidal effects and interactions between bound halos beyond the virial radii of locally dynamically dominant halos.
\end{abstract}

Key words: galaxies: evolution - galaxies: halos - galaxies: interactions

Online-only material: color figures

\section{INTRODUCTION}

Ongoing mergers of both dark matter halos and the galaxies they contain are an inevitable component of hierarchical structure formation. The potential impacts of these mergers on galaxy evolution are varied and heavily debated. Mergers can be roughly divided into two classes; minor mergers, in which a small halo is accreted by a substantially larger halo, and major mergers, in which the two halos are of roughly similar mass. The division between the two is usually placed near a mass ratio of 3:1. Minor mergers contribute both stars and gas to forming galaxies and are important for understanding the detailed morphologies of galaxies, particularly spiral galaxies. Tidal forces during a minor merger may heat the thin stellar disk and drive bar instabilities; thick disks may also represent the remnants of disrupted satellites (Steinmetz \& Navarro 2002; Yoachim \& Dalcanton 2008). The effects of a major merger are likely more dramatic. Major mergers between two gas-rich spiral galaxies are a popular mechanism for creating elliptical galaxies, an idea dating back to Toomre \& Toomre (1972). More recently, they have been invoked as a means of fueling intense starbursts and luminous active galactic nuclei (AGNs; Mihos \& Hernquist 1996; Di Matteo et al. 2005; Springel et al. 2005a, 2005b, among others).

The link between major mergers, starburst and AGN fueling, and galaxy morphology has been extensively studied using simulations and semi-analytic models (Mihos \& Hernquist 1996; Springel 2000; Kauffmann \& Haehnelt 2000; Wyithe

\footnotetext{
3 Lyman Spitzer Junior Fellow.
}

\& Loeb 2002, 2003; Naab \& Burkert 2003; Barnes 2004; Springel et al. 2005b; Di Matteo et al. 2005; Cox et al. 2006; Bower et al. 2006; Croton et al. 2006; Hopkins et al. 2006; Best et al. 2006; Fabian et al. 2006), and there is strong circumstantial observational evidence supporting the standard theory linking major mergers, AGNs, and galaxy evolution (Scoville et al. 1986; Sargent et al. 1987, 1989; Sanders \& Mirabel 1996; Magorrian et al. 1998; Ferrarese \& Merritt 2000; Gebhardt et al. 2000; Norton et al. 2001; McLure \& Dunlop 2002; Tremaine et al. 2002; Marconi \& Hunt 2003; Komossa et al. 2003; Gerssen et al. 2004; Yang et al. 2004; Goto 2005; Jogee 2005; Alexander et al. 2005; Borys et al. 2005; Owers et al. 2007). Theories connecting major mergers, starbursts, AGNs, and the creation of elliptical galaxies, however, have not been suitably tested observationally. Theoretical models include assumptions about AGN and starburst lifetimes and feedback efficiencies which tend to be under-constrained by the observational data. Additional constraints on these models are needed, as are model-independent observational tests of major merger driven evolutionary scenarios. Environment is a potentially powerful probe which has been under-exploited. To make use of environment, the environmental dependencies of the major merger rate must be well understood. This work therefore explores the environments of major mergers in the Millennium Simulation (MS), a large $N$-body simulation with a box length of $500 \mathrm{~h}^{-1} \mathrm{Mpc}$ and a particle mass below $10^{9} M_{\odot}$ (Springel et al. 2005c). This simulation is large enough to probe the full range of environments and has a fine enough mass resolution to follow galaxy-like halos and subhalos. The goal of this project is to provide the theoretical groundwork necessary 
to use environment as a probe of major merger driven galaxy evolution scenarios.

\subsection{Mergers and Environment: Previous Results}

The earliest, and simplest, theoretical studies of the major merger rate, $R_{\mathrm{mm}}$, were based on extended Press-Schechter theory, which was, in turn, based on linear theory plus spherical collapse models (Press \& Schechter 1974; Bond et al. 1991; Lacey \& Cole 1993). Following accretion, mergers between halos were assumed to occur on the timescale of dynamical friction, and mergers between subhalos were neglected. With these assumptions, merger trees can be built analytically for all halos existing today (Kauffmann \& White 1993; Somerville \& Kolatt 1999). The assumption that mergers occur on a dynamical friction timescale can also be applied to $N$-body simulations when subhalos cannot be resolved. Under this assumption, Kauffmann \& Haehnelt (2000) find that $R_{\mathrm{mm}}$ is independent of local environment. Extensions of this treatment first considered the merger rate between two subhalos, concluding that the sub-sub major merger rate could be quite high within group mass hosts. Further work began to include dynamical effects within the host halo such as tidal stripping. This was done both analytically and with $N$-body simulations (Mamon 2000; Peñarrubia \& Benson 2005; Boylan-Kolchin et al. 2008). Assuming that linear theory correctly describes accretion histories, all nonlinear effects are confined within host halos. Correlations between the major merger rate and environment on scales beyond the hosts' virial radii arise from convolving correlations between the specific $R_{\mathrm{mm}}$ and host properties and the hosts' environments. (For an example of this effect, see Collister \& Lahav 2005 or Blanton et al. 2007.) Linear theory breaks down when tidal forces become important, so some deviations should be expected.

It is attractive to study the physics of major mergers within host halos because doing so captures the majority of the nonlinear physics affecting $R_{\mathrm{mm}}$. Under the assumptions of linear theory, once a halo mass is specified, in this case the mass of the host halo, the accretion history is independent of environment (White 1996). Several recent studies of halo properties have indicated that accretion history does have a residual dependence on local environment (Wechsler et al. 2006; Croton et al. 2007; Gao \& White 2007). There may be a similar dependence between the specific major merger rate and local environment.

Within a bound virialized halo, where it may be possible to assume relative subhalo velocities are random, the specific major merger rate between subhalos can be expressed as

$$
R_{\mathrm{mm}}=n_{s}\left\langle\sigma_{m} v\right\rangle,
$$

where $\left\langle\sigma_{\mathrm{mm}} v\right\rangle$ is the major merger cross section averaged over the distribution of relative velocities and $n_{s}$ is the number density of potential major merger partners. Analytical studies that adopt a range of major merger cross sections and assume a velocity distribution for subhalos show that the major merger rate between subhalos decreases drastically with host halo mass while increasing with subhalo mass (Mamon 1992, 2000; Makino \& Hut 1997). For example, Mamon (2000) finds $R_{+} \propto n_{s} G^{2} m_{s}^{2} / V_{h}^{3}$, where $m_{s}$ is the subhalo mass and $V_{h}$ is the internal velocity of the host. These studies find that for subhalos selected on $m_{s}$ specific major merger rates are enhanced in groups and suppressed in clusters.
Analytical and numerical treatments both indicate that tidal stripping occurs on a much shorter timescale than dynamical friction, resulting in a population of tidally stripped subhalos for which dynamical friction is ineffective (Mamon 2000; Peñarrubia \& Benson 2005; Boylan-Kolchin et al. 2008). When a subhalo catalog is selected above a minimum mass, tidal stripping reduces the subhalo number densities by removing subhalos from the sample. In a subhalo sample based on preaccretion masses, tidal stripping can drastically reduce the subhalo major merger cross section. Previous work has taken the first approach.

Numerical simulations support the analytical results. In simulated clusters, the merger rate drops once subhalos are accreted (Ghinga et al. 1998; De Lucia et al. 2004). Gottlöber et al. (2001) make a complementary measurement in a cosmological $N$-body simulation. They measure the major merger rate of the most massive progenitors (MMPs) of the halos identified at $z=0$, therefore not counting major mergers whose remnants later merge with a more massive halo. They find that halos that reside in clusters at $z=0$ have the lowest major merger rates near $z=0$, but had major merger rates higher than the progenitors of isolated halos in the past. The major merger rates for halos that reside in groups at $z=0$ are higher than for isolated galaxies.

While the environments of major mergers are clearly a widely studied topic, the MS should allow us to make an important advancement. The MS, with its superb combination of size and resolution, allows the study of all of the above issues in concert. We use a common, well-defined language to study each issue and examine the interplay among them. We focus on studying the dynamics of major mergers and corresponding environmental signatures. Finally, we use definitions of environment and the major merger rate that have clear observational counterparts. This is essential as the ultimate goal is to use the results of this work to craft observational tests that are capable of identifying major merger populations.

We intend to use a dark matter simulation to develop environmental diagnostics of major merger populations that can be used to test the major merger driven galaxy evolution scenario. By doing so, we are assuming both a one-to-one correspondence between dark matter mergers and galaxy mergers, and that the relevant dynamics are dominated by the dark matter. When the cores of two dark matter halos that each host a galaxy merge, the galaxy merger is imminent (see the review in Barnes \& Hernquist 1992). The final stages of the galaxy merger occur quickly; Cox et al. (2006) find that the final galaxy merger in a 1:1 merger takes $\approx 200$ Myr. While there has been some discussion of "dark halos" which do not host galaxies (Maccio et al. 2006, and references therein), this occurs at $V_{\max }$ well below those considered here. Similarly, there is no observational evidence for orphan galaxies (Mandelbaum et al. 2006), and truncated dark matter halos have been observed around galaxies in clusters (Natarajan et al. 2007). A one-to-one correspondence between halo mergers and galaxy mergers is therefore a reasonable assumption. When subhalos begin to be artificially dissolved due to mass resolution, unphysical "orphan" galaxies may remain in the simulation (e.g., Kitzbichler \& White 2008). We avoid this issue by examining the effects of subhalo completion on our individual results. While the inclusion of baryons might affect some of the relevant dynamics, dark matter constitutes a strong majority of the matter. Hence, the dynamics is dominated by the dark matter, with possible refinements to be introduced by including baryons. Exploring this issue is a potential topic for future work. 


\section{METHODS}

\subsection{Simulation}

Our study is performed using the MS (Springel et al. 2005c). The MS is a cosmological $N$-body simulation of the $\Lambda$ CDM universe that follows the evolution of more than 10 billion dark matter particles in a box of $500 h^{-1} \mathrm{Mpc}$ comoving on a side. The particle mass is $8.6 \times 10^{8} h^{-1} M_{\odot}$, and particle-particle gravitational interactions are softened on scales smaller than $5 \mathrm{~h}^{-1} \mathrm{kpc}$. The simulation uses parameters in agreement with the WMAP1 results (Spergel et al. 2003): $\Omega_{m}=0.25, \Omega_{\Lambda}=0.75$, $h=0.73, n=1$, and $\sigma_{8}=0.9$. We use the publicly available halo catalog and merger trees which are described in the supplementary information to Springel et al. (2005c). Halos are identified in a two-step process in which particles separated by less than 0.2 times the mean particle separation are grouped together into a friends-of-friends (FOF) group, and SUBFIND (Springel et al. 2001) is then run on the FOF groups. A dominant "central" halo is identified in each FOF group, and all other halos are tagged as subhalos. The mean density in the FOF groups is somewhat lower than the expected overdensity of virialized halos at low $z$ and approaches the expected overdensity as $z$ increases. Bound structures with greater than 20 particles are kept in the halo catalog. Various properties are determined for each halo, among them are the maximum of the rotational velocity curve $\left(V_{\max }\right)$ and the mass that corresponds to the overdensity at which structures become nonlinear at each redshift. We take this "top hat" mass as the virial mass and use it to compute virial radii for host halos.

Motivated by our interest in subhalos, we characterize halos primarily by the maximum of their rotation velocity curve. SUBFIND may not provide reliable subhalo masses (Natarajan et al. 2007), and subhalo mass is itself a relatively ill-defined concept. Subhalos undergo substantial tidal stripping, but in the majority of cases the central galaxy remains intact. As a measure of the central halo potential, $V_{\max }$ is significantly better defined for subhalos. We are interested in using a halo property that correlates strongly with the luminosity of the galaxy hosted by the halo. Several recent studies make a case that $V_{\max }$ quantifies baryon's ability to cool within a halo and successfully compare $N$-body simulations to observations under this assumption (Kravtsov et al. 2004a; Tasitsiomi et al. 2004, 2008). The central region of a tidally stripped subhalo will relax and expand, lowering $V_{\max }$; hence $V_{\max }$ is not immune to the effects of tidal stripping (Hayashi et al. 2003; Kravtsov et al. 2004b; Klimentowski et al. 2009). The map between $V_{\max }$ and luminosity may also differ between central and subhalos. Despite the issues with $V_{\max }$, it is an improvement over using the tidal mass. We will try to focus on results that are relatively insensitive to these modeling issues and will consider their possible impact on our results.

\subsection{Merger Trees}

Each halo in the MS halo catalog is associated with a merger tree that contains all of the progenitors, and hence all of the mergers, of each halo in all previous simulation outputs. Tightly bound particles are given a higher weight when matching progenitors to descendants; hence, this scheme aims to track the inner cores of halos which are less vulnerable to mergers and tidal stripping. We identify "major" mergers within the publicly available merger trees using the $V_{\max }$ ratio, requiring $V_{\mathrm{LMP}} / V_{\mathrm{MMP}}>0.7$, where the progenitor with the higher $V_{\max }$ is referred to as the MMP, or most massive progenitor, and that with the lower $V_{\max }$ as the LMP, or least massive progenitor. In the case of halos that are not subhalos, $V_{\max }$ is closely correlated with mass, and this is the equivalent of mergers with less than a 3:1 mass ratio. For subhalos, the mass ratio of the halos may vary, but the stellar mass ratios should correspond to major mergers.

Two definitions of a specific major merger rate are used in this paper, where a specific major merger rate measures the number of major mergers per Gyr normalized by the number of halos, as opposed to a physical number density of major mergers per Gyr. The first rate is a backward-looking specific major merger rate, $R_{-}$, which is defined as the number of major mergers in the last time step that resulted in remnants with $V_{\max }$ divided by the number of halos with the same $V_{\max }$ in the current simulation output, $R_{-} \equiv N_{m-}\left(V_{\max }\right) / N_{h}\left(V_{\max }\right)$. That is, $R_{-}$measures the fraction of halos that are a remnant of a major merger that occurred in the last Gyr. The observational equivalent of this definition is a specific major merger rate measured using morphologically identified major merger remnants. It is also the appropriate rate to compare with potential post-major merger populations such as starbursts and luminous AGNs. A forward-looking specific major merger rate, $R_{+}$, is defined as the number of halos in the $V_{\max }$ range of interest that will experience a major merger in the next time step divided by the number of halos in the same $V_{\max }$ range in the current time step, $R_{+} \equiv N_{m+}\left(V_{\max }\right) / N_{h}\left(V_{\max }\right)$. The forward-looking specific major merger rate measures the fraction of halos that will become the MMP of a major merger in the next Gyr. The observational equivalent of $R_{+}$is a specific major merger rate measured using pair counts. In these definitions, $N_{m-}$ and $N_{m+}$ distinguish major mergers that happened in the previous Gyr from those that will happen in the next Gyr.

\subsection{Numerical Issues}

The results presented here are sensitive to the completion of the halo catalog, which includes both distinct halos and subhalos. We work with a subset of the full MS halo catalog which includes halos with $V_{\max } \geqslant 120 \mathrm{~km} \mathrm{~s}^{-1}$, though our major mergers may have progenitor halos with $V_{\max }$ down to $\approx 100 \mathrm{~km} \mathrm{~s}^{-1}$. The complete halo catalog includes all halos with a bound mass greater than $1.7 \times 10^{10} M_{\odot}$. We test for a flattening of the $V_{\max }$ function in our halo catalog and find no sign of incompletion for isolated halos down to $V_{\max }=120 \mathrm{~km} \mathrm{~s}^{-1}$ at $z=0$ or $z=4$. While $M_{v}$ scales as $V_{\max }^{3}$ at all redshifts, the virial mass at fixed $V_{\max }$ decreases with redshift. At redshift $z=0$, we expect an average distinct halo with $V_{\max }=100 \mathrm{~km} \mathrm{~s}^{-1}$ to have a virial mass $M_{v} \approx 1.2 \times 10^{11} M_{\odot}$ and include $n_{p} \approx 150$ bound particles. At $z=4$, an average distinct halo with $V_{\max }=100 \mathrm{~km} \mathrm{~s}^{-1}$ has a virial mass $M_{v} \approx 3.3 \times 10^{10} M_{\odot}$ and includes $n_{p} \approx 38$ bound particles. While the halo catalog appears to be complete for isolated halos at $z=4$, completion may be an issue at high $z$ for major merger remnants which require the retention of halos down to $100 \mathrm{~km} \mathrm{~s}^{-1}$. In general, the effects of halo completion on our results are redshift dependent.

Subhalos may suffer from over-merging if inadequate mass and force resolution allow subhalos to be prematurely disrupted. As a subhalo is stripped and the number of bound particles decreases, two-body interactions can cause a subhalo to artificially evaporate, hence the mass resolution can limit the mass, or $V_{\max }$, to which subhalos can be reliably identified. Similarly, inadequate force resolution results in subhalos with artificially large cores which are easily disrupted and artificially lost. While ideally one would rerun the MS with increased mass and spatial 

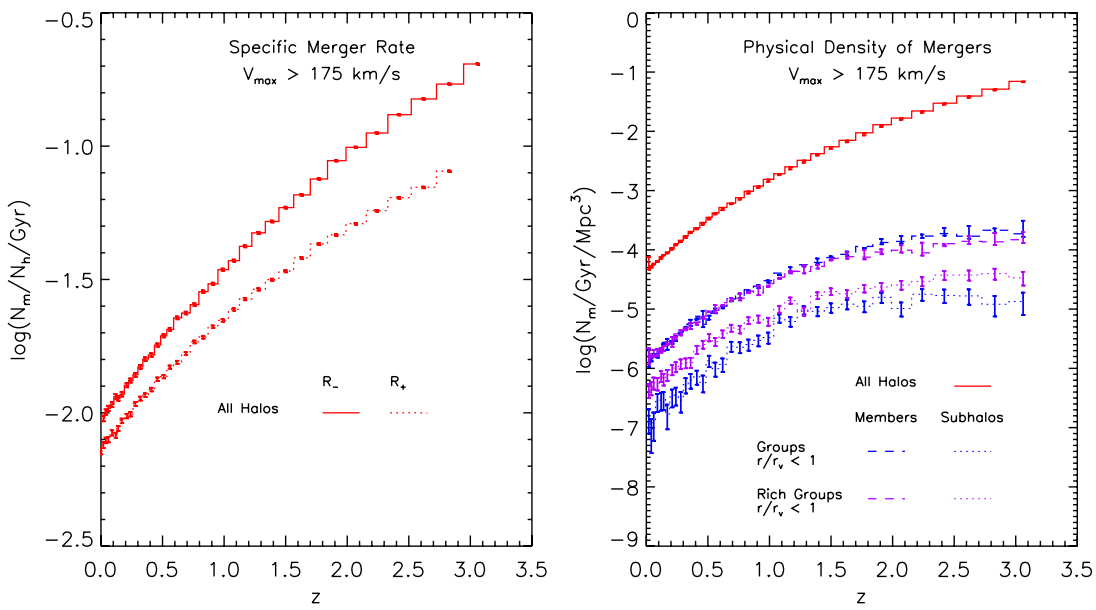

Figure 1. Evolution of the major merger rate, $R_{\mathrm{mm}}$. The left panel displays the evolution of the specific $R_{\mathrm{mm}}$, the major merger rate per halo, for all halos with $V_{\max } \geqslant 175 \mathrm{~km} \mathrm{~s}^{-1}$. The forward-looking rate, $R_{+}$, and the backward-looking rate, $R_{-}$, are both plotted. These rates are defined in the text. The right panel displays the physical number density of major mergers per Gyr resulting in remnants with $V_{\max } \geqslant 175 \mathrm{~km} \mathrm{~s}^{-1}$. The (red) solid line corresponds to all halos. The (blue and purple) dashed and dotted lines correspond to major mergers occurring in "groups" and "rich groups" (see the text for definitions). The dashed lines include both sub-sub and host-sub major mergers, and the dotted lines include only sub-sub major mergers.

(A color version of this figure is available in the online journal.)

resolution to estimate the impact of these effects, this route is prohibitively expensive. These issues have initiated multiple numerical studies, and as an alternative to rerunning the simulation we will rely on the results of Klypin et al. (1999), which agree with other, similar, numerical studies. Klypin et al. (1999) provide a pair of criteria that must be fulfilled in order for over-merging of subhalos not to be important. To avoid over-merging, subhalos must retain 20-30 particles post-tidal stripping and the tidal radius must be at least as large as a couple of spatial resolution lengths. These limits are not exact, but they highlight the relevant concerns and will be used to indicate approximately when subhalo completion becomes an issue. The possible effects of subhalo completion on our individual results are discussed in detail in Section 4.3. We favor results that are relatively insensitive to subhalo incompletion.

The final simulation issue which must be considered is the spacing of the simulation outputs for which the halo finder is run. There are 64 saved outputs, most of which are equally spaced in $\log (1+z)$ between $z=20$ and $z=0$. The typical time elapsed between two consecutive outputs is $2-400 \mathrm{Myr}$. Typical timescales for an accreted halo to merge with its host halo are a few to $10 \mathrm{Gyr}$ (Boylan-Kolchin et al. 2008), though we find that in some cases this timescale may be shortened by approximately a factor of 10 . Therefore, except in some extreme cases, accreted halos are resolved as subhalos in multiple outputs before a merger with the host occurs. This temporal resolution distinguishes this work from some past studies (e.g., Klypin et al. 1999).

\section{RESULTS}

This section presents the evolution of $R_{\mathrm{mm}}$ and several correlations between $R_{\mathrm{mm}}$ and environment. While each measurement is motivated physically, a full discussion is postponed until Section 4. In what follows, a "subhalo" resides within the virial radius of its host halo, and a "distinct" halo is any halo that is not a subhalo. A distinct halo may host subhalos, and therefore be a "host" halo. While $V_{\max }$ may be used to designate the maximum circular velocity of any halo, $V_{h}$ is used specifically for either distinct or host halos and $V_{s}$ specifically for subhalos.

\subsection{Evolution of the Merger Rate}

Figure 1 presents the evolution of $R_{\mathrm{mm}}$ for all halos with $V_{\max } \geqslant 175 \mathrm{~km} \mathrm{~s}^{-1}$. The left panel displays the evolution of both specific major merger rates, $R_{+}$and $R_{-}$, and the right panel the evolution of the physical density of mergers. The backward major merger rate, $R_{m-} \propto(1+z)^{2.20 \pm 0.05}$ and the forward major merger rate, $R_{m+} \propto(1+z)^{1.82 \pm 0.01}$. Due to completion issues at high $z$, the actual slopes may be somewhat steeper. The two specific major merger rates differ by the $V_{\max }$ of the halos which are used to compute the specific $R_{\mathrm{mm}}$ from the absolute $R_{\mathrm{mm}}$, that is $R_{-} / R_{+} \approx N_{h}\left(V_{\mathrm{MMP}}\right) / N_{h}\left(V_{\mathrm{rem}}\right)$. The evolution of the $V_{\max }$ function accounts for the difference between the two slopes. The evolution of the specific major merger rate depends on whether it is defined using the simulation equivalent of pair counts or of merger remnants.

The physical number density of major merger remnants with $V_{\max } \geqslant 175 \mathrm{~km} \mathrm{~s}^{-1}$ increases with redshift like $n_{m} \propto$ $(1+z)^{5.44 \pm 0.02}$. For comparison, the physical number density of halos with $V_{\max } \geqslant 175 \mathrm{~km} \mathrm{~s}^{-1}$ evolves as $n_{h} \propto(1+z)^{3.13 \pm 0.03}$. The evolution of the physical densities differs from their massselected counterparts due to the evolution of the $V_{\max }-$ mass relation. This is most notable at high $z$ where halos in a massselected halo sample may become scarce, but halos in a $V_{\max }{ }^{-}$ selected sample simply correspond to lower mass halos. In a similar fashion, measuring the evolution observationally is complicated by any evolution in the mass or $V_{\max }$ estimator used to define the galaxy sample.

\subsection{Mergers Between Subhalos and Their Host Halos}

When a halo is accreted by a more massive host halo and becomes a subhalo, it is potentially subject to tidal stripping, dynamical friction, unbound and bound collisions with other subhalos, and may eventually merge with the host halo. The results presented in the following two subsections examine these issues in the light of their influence on $R_{\mathrm{mm}}$.

Dynamical friction is not effective at degrading the orbits of tidally stripped subhalos (Boylan-Kolchin et al. 2008). To study tidal stripping in the MS, we define the measure $n_{\mathrm{bp}} / n_{\mathrm{bp}}\left(V_{\mathrm{max}}\right)$, where $n_{\mathrm{bp}}$ is the number of particles that remain bound to the 

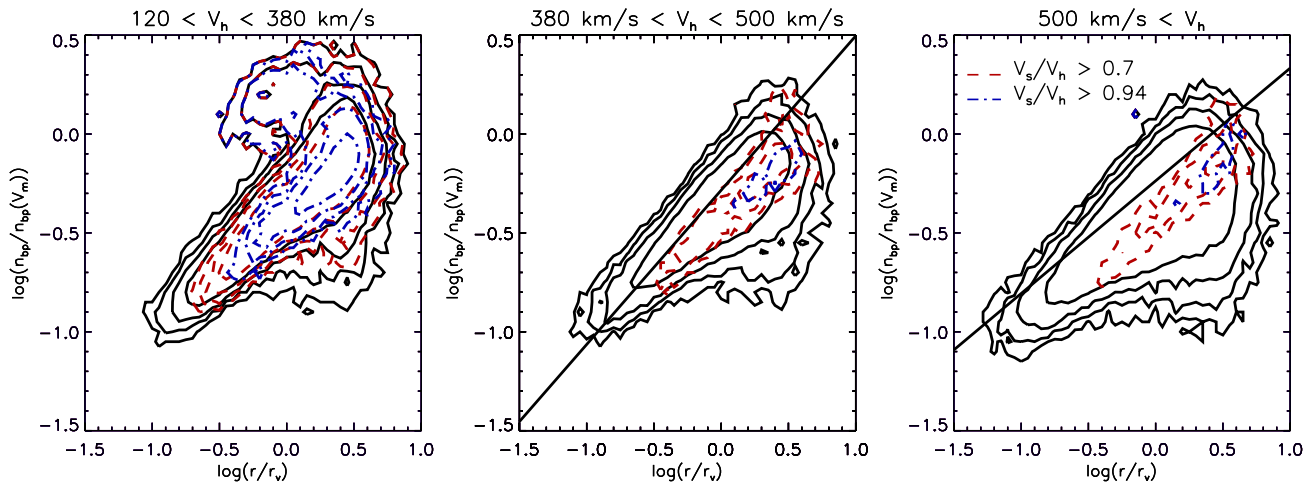

Figure 2. Tidal stripping of subhalos at $z=0$. Logarithmically, spaced contours of subhalo number density in the $\log \left(n_{\mathrm{bp}} / n_{\mathrm{bp}}\left(V_{\mathrm{max}}\right)\right)$ vs. $\log \left(r_{o} / r_{v h}\right)$ plane, where $n_{\mathrm{bp}} / n_{\mathrm{bp}}\left(V_{\mathrm{max}}\right)$ is a well-defined measure of tidal stripping which is discussed in the text and $r_{o} / r_{v h}$ is the subhalo's distance from the center of the host halo in units of the host halo's virial radius. The three panels are for subhalos residing in hosts with $V_{h}$ values corresponding to galaxy-like host halos, group-like host halos, and rich group and cluster-like host halos. The black contours are for all subhalos with $V_{s}>120 \mathrm{~km} \mathrm{~s}^{-1}$. The (red) dashed and (blue) dot-dashed contours are subhalos whose merger with the host halo would be counted as a major merger. Contours are shown for $V_{s} / V_{h}>0.7$ and $V_{s} / V_{h}>0.94$, roughly corresponding to pre-tidal stripping mass ratios of 1:3 and 1:1.2. The thin diagonal lines show the predicted relationships between $m_{\mathrm{ts}} / m_{\mathrm{vs}}$ and $r_{\text {peri }} / r_{\mathrm{vh}}$ for NFW halos for group-like (center panel) and cluster-like (right panel) hosts from Mamon (2000), where $r_{\text {peri }}$ is the pericenter of the subhalo's orbit.

(A color version of this figure is available in the online journal.)
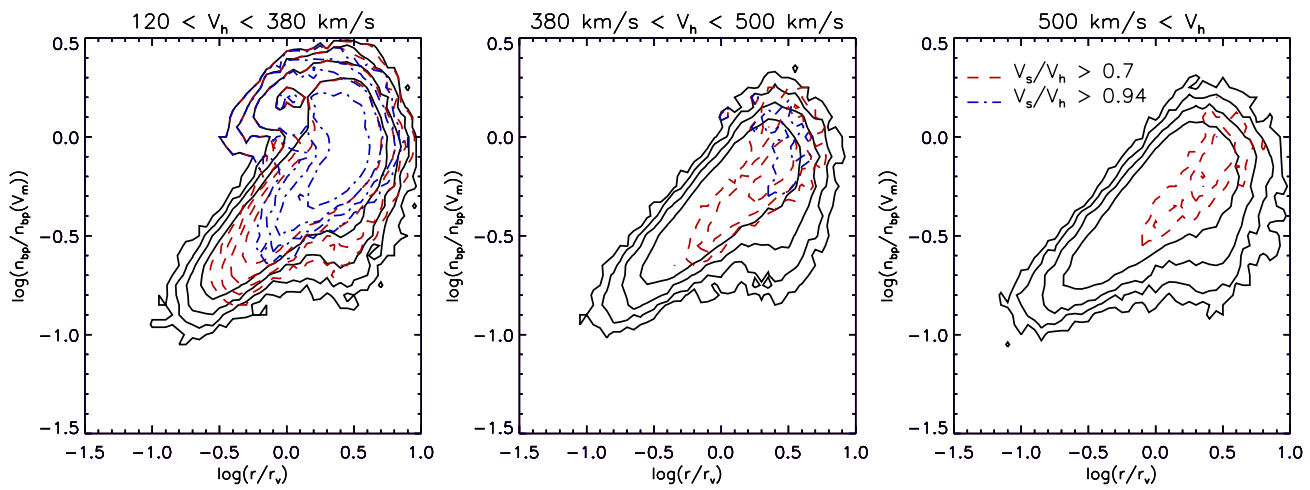

Figure 3. Tidal stripping of subhalos at $z=1$. The same as Figure 2, but for $z=1$.

(A color version of this figure is available in the online journal.)

subhalo and $n_{\mathrm{bp}}\left(V_{\max }\right)$ is the typical number of bound particles in a distinct halo with the same $V_{\max }$. Distinct halos exhibit a tight correlation between $n_{\mathrm{bp}}$ and $V_{\max }$ with small but measurable scatter, as expected, and $\log \left(n_{\mathrm{bp}} / n_{\mathrm{bp}}\left(V_{\mathrm{max}}\right)\right)$ is therefore well defined. This measure is selected to probe tides. While $V_{\max }$ is a measure of the current depth of a subhalo's central potential, $n_{\text {bp }}$ is the number of particles currently bound to the subhalo, $\epsilon<0$, not the number of particle within a tidal radius.

Figures 2 and 3 demonstrate tidal stripping in the MS. They show subhalo number density contours in the $\log \left(n_{\mathrm{bp}} / n_{\mathrm{bp}}\left(V_{\mathrm{max}}\right)\right)$ versus $r_{o} / r_{\mathrm{vh}}$ plane at $z=0$ and $z=1$, where $r_{0} / r_{\mathrm{vh}}$ is the current orbital radius of the subhalo divided by the virial radius of the host halo. As the central potential responds to mass loss, contours in this plane likely show a tighter correlation than in the $m_{\mathrm{ts}} / m_{\mathrm{vs}}$ versus $r_{o} / r_{\mathrm{vh}}$ plane, where $m_{\mathrm{ts}}$ and $m_{\mathrm{vs}}$ are the tidal mass and pre-accretion virial mass of the subhalo. Contours are shown for all subhalos, and for subhalos with $V_{s} / V_{h}>0.7$ and 0.94 , corresponding to approximate, pre-accretion, mass ratios of $3: 1$ and nearly $1: 1$ (1:1.2). The three panels show subhalos residing in galaxy-like host halos, $175<V_{h}\left(\mathrm{~km} \mathrm{~s}^{-1}\right)<380$, group-like host halos, $380<V_{h}\left(\mathrm{~km} \mathrm{~s}^{-1}\right)<500$, and a combination of rich grouplike host halos, $500<V_{h}\left(\mathrm{~km} \mathrm{~s}^{-1}\right)<950$ and cluster-like host halos, $950<V_{h}\left(\mathrm{~km} \mathrm{~s}^{-1}\right)$. Later "group-like" and "cluster-like" host halos will be distinguished from halos that actually host groups and clusters. Figures 2 and 3 include "subhalos" from the FOF groups that are beyond the virial radius of the central host halo.

In Figures 2 and 3, it can be clearly seen that subhalos with $V_{s} / V_{h}$ values that for distinct halos would correspond to $3: 1$ and lower mass ratios are accreted and effectively tidally stripped by the host halo. The exception is the case of subhalos with $V_{s} / V_{h}$ values near 1:1 which show a spur at high $\log \left(n_{\mathrm{bp}} / n_{\mathrm{bp}}\left(V_{\max }\right)\right)$ within the virial radius of the host. Excluding this spur, the density contours for potential major merger progenitors lie above the densest contours for all halos, consistent with a small random subset of all halos. While these subhalos are found primarily at large radii, there is no significant change in the distribution of $V_{s} / V_{h}$ with $r_{o} / r_{\mathrm{vh}}$.

The vast majority of major mergers can be cleanly separated into host-sub and sub-sub mergers; that is, "host-host" mergers are rare. Figure 4 shows the distribution of $V_{\mathrm{LMP}} / V_{\mathrm{MMP}}$ for major mergers resulting in galaxy-like, group-like, and rich groupand cluster-like remnants at $z=0,1$, and 2. Major mergers are dominated by mergers with $V_{\mathrm{LMP}} / V_{\mathrm{MMP}}<0.94$. Only a few percent of major mergers are close to 1:1 mergers. Major mergers are therefore occurring in the regime in which the LMP is accreted as a subhalo and tidally stripped before merging with its host halo.

Figure 5 presents both $R_{+}$(left panel) and $R_{-}$(right panel) for host-sub major mergers resulting in group-like halos. This mass regime is chosen to limit the effects of subhalo completion on 

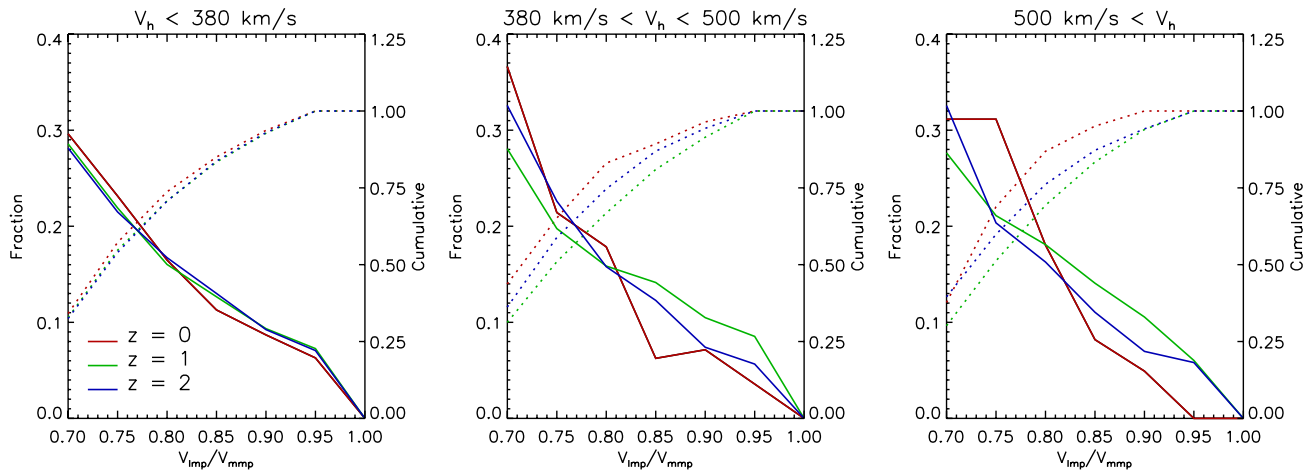

Figure 4. Distribution of the circular velocity ratio between the least and most massive progenitors, $V_{\mathrm{LMP}} / V_{\mathrm{MMP}}$, for major mergers at $z=0$, 1 , and 2 , split by merger remnant $V_{\max }$ as indicated. The solid lines show the fractional distribution and the dashed lines the cumulative probability. These distributions are coarsely binned; hence the cumulative probability distribution starts above 0 .

(A color version of this figure is available in the online journal.)
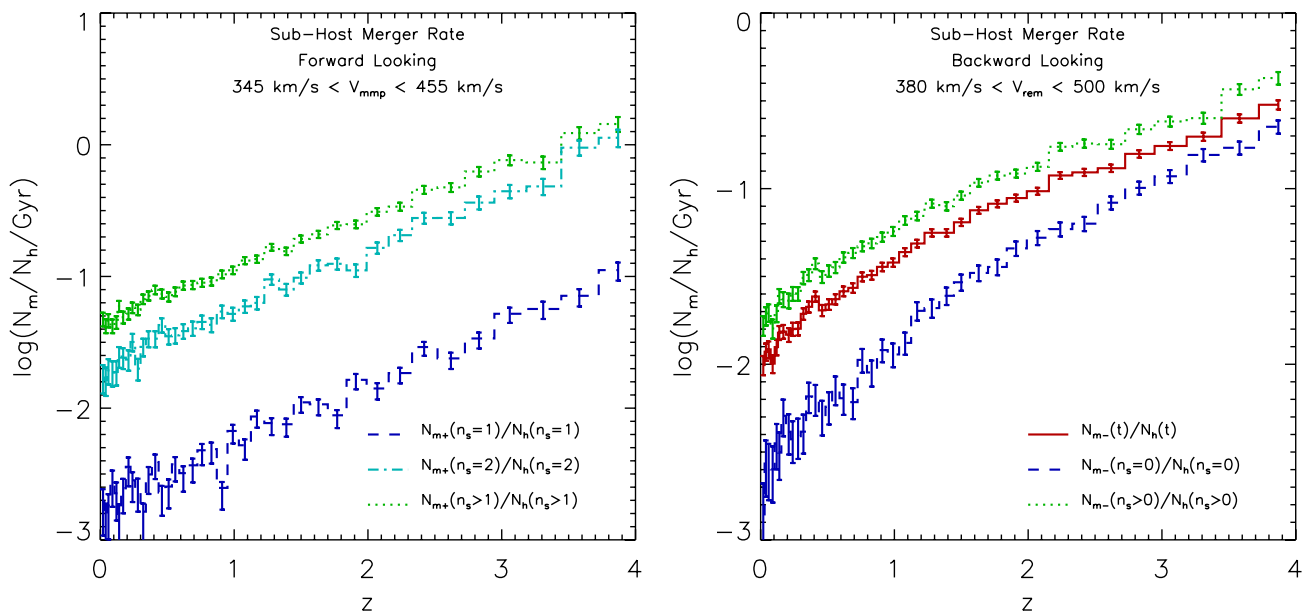

Figure 5. Effect of interactions between subhalos on the specific major merger rate. The left panel shows $R_{+}$for host-sub mergers in hosts with $345<V_{h}\left(\mathrm{~km} \mathrm{~s}^{-1}\right)<$ 455 split by the number of subhalos in the host. The (dark blue) long-dashed line shows $R_{+}$for mergers between a host and a lone subhalo. The (light blue) dot-dashed line shows $R_{+}$for host-sub mergers of hosts with two subhalos, and the green dotted line shows $R_{+}$for host-sub mergers of hosts with at least two subhalos. The right panel shows $R_{-}$for group-like halos, which are the typical remnants of the mergers in the left panel. The (dark blue) dashed line shows $R_{-}$for halos with no subhalos with $V_{s}>120 \mathrm{~km} \mathrm{~s}^{-1}$. These remnants would be the result of major mergers between the host and a single bright subhalo. The (green) dotted line shows $R_{-}$among group-like halos with at least one bright subhalo and the (red) solid line shows $R_{-}$among all group-like halos.

(A color version of this figure is available in the online journal.)

our results. We expect the effectiveness of dynamical friction to be reduced by tidal stripping; however, $R_{+}$for host-sub major mergers can reach values as high as a major merger every one to a few Gyr, as can be seen in the left panel of Figure 5. This suggests additional dynamics are at play. In addition to interacting with their host, subhalos interact with each other, motivating us to examine whether the presence of multiple subhalos affects the host-sub specific major merger rate.

In the left panel of Figure 5, $R_{+}$is plotted for host halos with $345<V_{\max }\left(\mathrm{km} \mathrm{s}^{-1}\right)<455$ and 1,2, and greater than 1 subhalo, $n_{s}=1,2$, and $>1$. The subhalo counts include all subhalos with $V_{\max } \geqslant 120 \mathrm{~km} \mathrm{~s}^{-1}$. For comparison, a potential LMP subhalo in a host with $V_{h}=345 \mathrm{~km} \mathrm{~s}^{-1}$ has $V_{s} \geqslant 210 \mathrm{~km} \mathrm{~s}^{-1}$. To compute the $n_{s}$ dependent $R_{+}$, at each time step, $t$, the number of new major merger remnants at $t+1$ with $n_{s}-1$ subhalos and an MMP with $345<V_{\max }\left(\mathrm{km} \mathrm{s}^{-1}\right)<455$ is divided by the number of halos at $t$ with $n_{s}$ subhalos and $345<V_{\max }\left(\mathrm{km} \mathrm{s}^{-1}\right)<455$. The right panel of Figure 5 shows $R_{-}$for remnants with $380<V_{\max }\left(\mathrm{km} \mathrm{s}^{-1}\right)<500$, roughly corresponding to the remnants of the mergers plotted in the left-hand panel. To compute the $n_{s}$ dependent $R_{-}$, the number of major merger remnants with $n_{s}$ is divided by the number of all halos with $n_{s}$ in the same time step. Both rates are shown as $R_{+}$is easiest to interpret dynamically and $R_{-}$is easiest to compare with observations. Particularly in groups, an $R_{\mathrm{mm}}$ that relies on identifying major merger remnants rather than counting close pairs is easier to measure observationally. The differences between the two panels of Figure 5 arise from the choice of halo population with which $R_{\mathrm{mm}}$ is normalized, e.g., the number of hosts with $n_{s}=1$ versus $n_{s}=2$.

The specific forward-looking $R_{\mathrm{mm}}$ in host halos that have two subhalos is approximately 10 times $R_{+}$in host halos with only one subhalo pre-merger, and a similar trend is observed for $R_{-}$. The fraction of group-like host halos with $n_{s}>0$ that are major merger remnants is several times that of halos that do not host a subhalo with $V_{\max } \geqslant 120 \mathrm{~km} \mathrm{~s}^{-1}$. At low redshifts, which are less affected by subhalo incompletion, a second subhalo is present for $80 \%-90 \%$ of the host-sub major mergers in grouplike halos.

The measured trends cannot be attributed to a correlation between $n_{s}$ and $V_{s} / V_{h}$ or to our definitions of $n_{s}$ dependent $R_{\mathrm{mm}}$. Within the narrow range of $V_{h}$ considered, a host halo with two subhalos is only twice as likely to host a potential major merger LMP. Our definitions of $n_{s}$ dependent $R_{\mathrm{mm}}$ assume, for 
example, that all major merger remnants with no subhalos are the result of a merger between a host and its single subhalo. When a lone host-sub merger and the accretion of a separate subhalo occur simultaneously in the same host, it is falsely identified as a merger for which a subhalo was present. As there are $\approx 2.5$ times as many halos with one subhalo as with two (at $z=0$ ), such misidentifications could bias our results. The small time step between outputs significantly mitigates this effect. To bring the true ratio of merger rates $\left(R_{+}\right)$down to 2 would require an accretion rate much greater (a factor of $\sim 100$ ) than that found in the MS. This concern does not affect $R_{-}$as both remnants and the halo counts used to normalize $R_{-}$are identified in the same time step.

A physical mechanism for enhancing the host-sub $R_{\mathrm{mm}}$ in hosts with multiple subhalos is advanced in Section 4.1.

\subsection{Mergers Between Subhalos}

Previous theoretical studies concluded that $R_{\mathrm{mm}}$ is enhanced in groups when subhalos are selected above a minimum mass. This section improves upon such studies by considering a subhalo sample selected above a minimum $V_{\max }$. Our focus must be on sub-sub mergers as subhalos dominate numerically in groups by definition. While host-sub major mergers are enhanced in "groups," we find sub-sub mergers are suppressed compared to the major merger rate for distinct halos.

Observationally defined groups are an attempt to identify bound virialized objects by finding overdensities in the galaxy redshift space distribution. The dark matter simulation equivalent is a host halo and its bound subhalos. A defining characteristic of "groups" is the presence of multiple galaxies. Accordingly, groups in the simulation are defined as group-like halos, $380<V_{h}\left(\mathrm{~km} \mathrm{~s}^{-1}\right)<500$, with $n_{s}>2$, where $n_{s}$ is again the number of subhalos with $V_{\max }>120 \mathrm{~km} \mathrm{~s}^{-1}$ within the host's virial radius. Rich groups and clusters are similarly required to have $n_{s}>2$. The observational equivalent would be a catalog of groups containing at least three members, the central galaxy and two satellites, selected above the luminosity equivalent of $V_{\text {max }}>120 \mathrm{~km} \mathrm{~s}^{-1}$.

Group members account for a small percentage of all major mergers, and sub-sub mergers account for $\sim 10 \%$ of major mergers in groups and a fraction of a percent of all major mergers. This is illustrated in the right panel of Figure 1, which displays the physical number densities of major mergers of all group members and of major mergers between two subhalos. The sub-sub $R_{\mathrm{mm}}$ shown is for subhalos in groups and rich groups, as indicated.

Figure 6 displays $R_{-}$versus redshift for halos with $V_{\max }>$ $120 \mathrm{~km} \mathrm{~s}^{-1}$ as a function of group membership and subhalo status for all halos, group members ("group members, $r / r_{v}<$ 1 "), members of rich groups ("rich group members, $r / r_{v}<$ 1 "), and sub-sub mergers in groups and rich groups. An alternate group definition based on the FOF groups, groups have $n_{s}>2$ subhalos within the FOF group and members include all subhalos within the FOF group, is also shown ("group members, FOF"). In a similar fashion, observational group finding algorithms vary in how conservatively they define groups and group members.

The $R_{\mathrm{mm}}$ for group members is suppressed and major mergers are dominated by host-sub mergers. Not only are major mergers between subhalos rare, but also the frequency of major merger remnants among subhalos is low. Subhalos display an $R_{-}$which is a factor of a few lower than for all halos, which are themselves dominated by distinct halos. Accordingly, $R_{-}$for all group

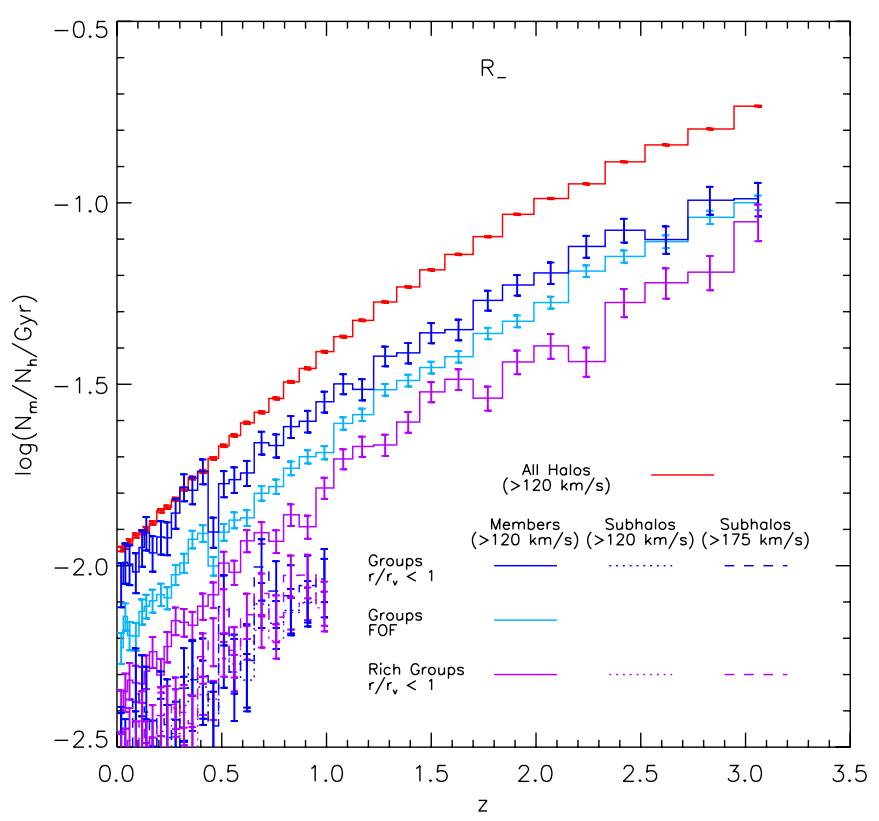

Figure 6. Specific major merger rate $R_{-}$for "group" and "rich group" members and subhalos (see definitions in the text). Groups have been defined using only subhalos with $r / r_{v} \leqslant 1$ and all "subhalos" in an FOF group. "Members" includes both central and subhalos. Subhalos include only major merger remnants that are subhalos. The (red) solid line shows $R_{-}$for all halos with $V_{\max } \geqslant 120 \mathrm{~km} \mathrm{~s}^{-1}$. The (dark blue, light blue, and purple) solid lines show $R_{-}$for all group members. The dotted (dashed) lines correspond to subhalos with $V_{\max } \geqslant 120 \mathrm{~km} \mathrm{~s}^{-1}\left(V_{\max } \geqslant 175 \mathrm{~km} \mathrm{~s}^{-1}\right)$.

(A color version of this figure is available in the online journal.)

members taken together is suppressed compared to distinct halos. We still see this suppression when only subhalos with $V_{s}>175 \mathrm{~km} \mathrm{~s}^{-1}$, which are less affected by incompletion, are considered. As the ratio of subhalos to host halos increases, moving from conservatively defined groups to FOF groups to rich groups, $R_{-}$for group "members" decreases.

Major merger remnants are somewhat more common among subhalos within host halos with $V_{h}<380 \mathrm{~km} \mathrm{~s}^{-1}$. This occurs most frequently for subhalos with $V_{s} / V_{h} \approx 1$, likely corresponding to a host-sub merger in disguise.

We attempted to measure a correlation between $R_{+}$and either $V_{h}$ or $V_{s}$, as predicted by analytical models. We measured no correlation with either. A similar result for $R_{-}$can be seen in Figure 6. We constrained this study to subhalos with $V_{\mathrm{MMP}}=V_{s} \geqslant 175 \mathrm{~km} \mathrm{~s}^{-1}$, for which we expect the LMPs to survive numerical effects at low redshift. The least massive LMPs have $V_{s} \approx 120 \mathrm{~km} \mathrm{~s}^{-1}$, the lower limit of our catalog. As regards $V_{h}$, the average $n_{s}$ scales as $V_{h}^{3}$, as assumed previously, but at the low $n_{s}$ present in our sample $\left\langle n_{s}^{2}\right\rangle \neq\left\langle n_{s}\right\rangle^{2}$, in conflict with the assumption of the analytical models. Attempting to restrict the analysis to $V_{h}$ with $\left\langle n_{s}\right\rangle>2$ resulted in a $V_{h}$ range which was too narrow and restricting the analysis to any host with $n_{s}>2$ resulted in very poor statistics at low $V_{h}$. A higher resolution simulation is needed to address this question.

In the high $V_{h}$ hosts, we measured no correlation between the sub-sub $R_{+}$and $V_{s}$. This is different than stating that there is no correlation with subhalo mass. While subhalo mass and $V_{s}$ are correlated, there is significant scatter in the mass $-V_{s}\left(\right.$ or $\left.n_{b p}-V_{s}\right)$ relation. Similarly, $V_{s}$-defined major mergers differ from massdefined major mergers. It should also be noted that the statistics are poor due to the low number of sub-sub mergers, and a weak trend with $V_{s}$ cannot be ruled out. 

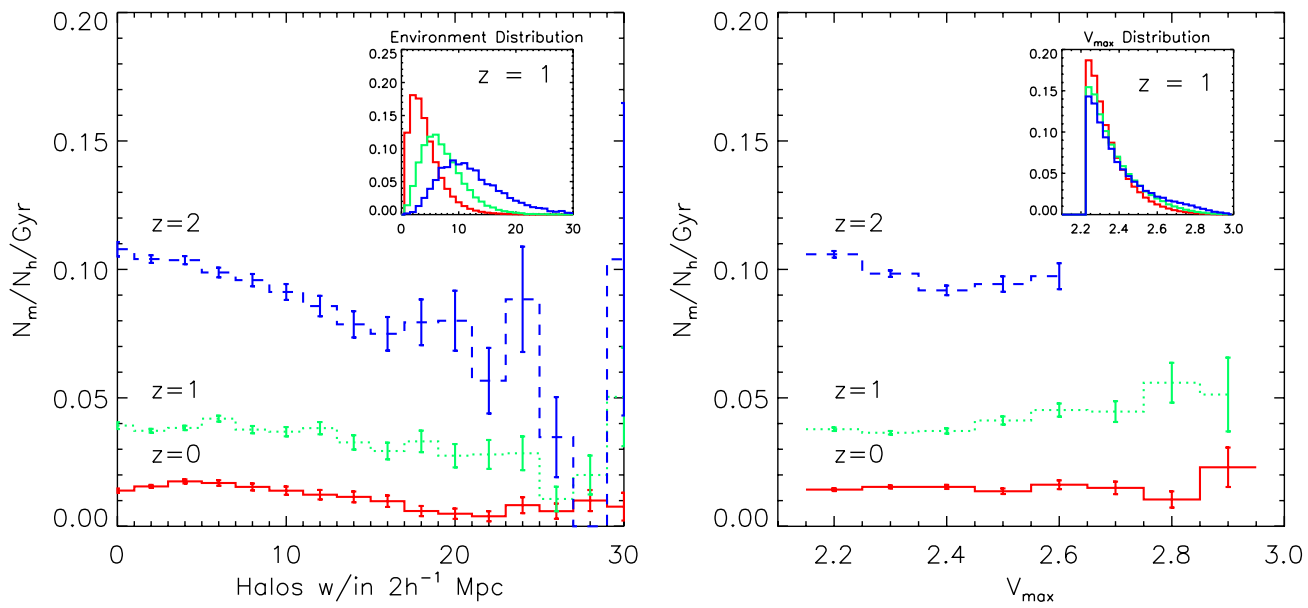

Figure 7. Major merger rate $R_{-}$vs. local halo environment, as defined in the text, and $V_{\max }$. The left panel displays $R_{-}$vs. the local halo environment for all halos with $V_{\max } \geqslant 175 \mathrm{~km} \mathrm{~s}^{-1}$ for $z=0,1$, and 2. The right panel shows $R_{-}$vs. $V_{\max }$ for halos in all environments. Insets are for orientation. The left inset shows the distribution of local densities for $z=1$ for three $V_{\max }$ ranges, galaxy-like $\left(175<V_{\max }\left(\mathrm{km} \mathrm{s}^{-1}\right)<380\right)$, group-like $\left(380<V_{\max }\left(\mathrm{km} \mathrm{s}^{-1}\right)<500\right)$, and rich group-like $\left(500<V_{\max }\left(\mathrm{km} \mathrm{s}^{-1}\right)<950\right)$. The right inset shows the distribution of $V_{\max }$ in three environment ranges, $0-4,5-9$, and $10-14$ halos within $2 h^{-1} \mathrm{Mpc}$ comoving.

(A color version of this figure is available in the online journal.)

\subsection{Merger Rate Versus Local Halo Environment}

In order to explore the relationship between the major merger rate and the environment beyond the virial radii of the host halos, we define the "local halo environment" as the count of all halos, both distinct and sub, with $V_{\max } \geqslant 120 \mathrm{~km} \mathrm{~s}^{-1}$ within a sphere of radius $2 h^{-1} \mathrm{Mpc}$ comoving centered on each halo. As progenitor identification is not an issue, subhalos are complete to lower $V_{\max }$ than merger remnants. Accordingly, we can use $V_{\max } \geqslant 120 \mathrm{~km} \mathrm{~s}^{-1}$ to measure environment, which has the advantage of sampling the environment more accurately.

We seek an environmental measure with an observable counterpart that is well correlated with the dark matter overdensity, which is likely the preferred measure when studying dynamics. This motivates us to include both distinct and subhalos so that the environment is weighted by both the mass and proximity of any nearby host halos. A halo near the edge of the $2 h^{-1} \mathrm{Mpc}$ sphere contributes to the local environment by as many of its subhalos as lie within the sphere. This also avoids the observational challenge of separating central and satellite galaxies.

Figure 7 shows $R_{-}$versus the local halo environment for all halos with $V_{\max } \geqslant 175 \mathrm{~km} \mathrm{~s}^{-1}$ in the left panel, and in the right panel displays $R_{-}$versus $V_{\max }$ for all halos in all environments. Both are shown for $z=0,1$, and 2 and the insets are provided for orientation. A correlation between $R_{-}$and either the local halo environment or $V_{\max }$ would be straightforward to compare with observations. Unfortunately, no such correlation is seen. While $R_{-}$declines slightly above local halo counts of 15-20 at $z=0$ and 1 , and a sharper decline is seen at $z=2$, the vast majority of halos reside in the less dense environments where no correlation is seen.

Recent work has revealed codependent correlations between halo properties such as concentration or age and both $V_{\max }$, or mass, and local environment in large $N$-body simulations (Gao et al. 2005; Wechsler et al. 2006; Croton et al. 2007; Gao \& White 2007; Wetzel et al. 2007). In Figure $8, R_{-}$is plotted versus the local halo environment for $z=0,1$, and 2 and $175<V_{\max }\left(\mathrm{km} \mathrm{s}^{-1}\right)<280,280<V_{\max }\left(\mathrm{km} \mathrm{s}^{-1}\right)<380$, and $380<V_{\max }\left(\mathrm{km} \mathrm{s}^{-1}\right)<900$. These $V_{\max }$ ranges were selected to probe the evolution of these trends. They correspond to regions in the $V_{\max }$ function at $z=0$ safely within the power-law component, approaching the turnover, and beyond the turnover.
For this figure, three simulation outputs were stacked at each redshift in order to improve the merger statistics for high $V_{\max }$ halos. The left panel includes all distinct halos while the right includes only the central halos of the FOF groups. Linear theory applies directly to distinct halos. The right panel is included because the dynamics of the central halos may differ from those of all distinct halos.

When halos are separated by $V_{\max }, R_{-}$correlates with local environment. For halos with the lowest values of $V_{\max }, R_{-}$for distinct halos decreases slightly with local halo environment, though only at atypically high environments. This is similar to the trend for all halos, which are dominated by halos with low $V_{\max }$. For the halos with higher $V_{\max }, R_{-}$increases significantly with local halo environment.

At this stage, it is prudent to examine whether the correlation between the number of subhalos in a host and $R_{-}$is responsible for the observed trend with local environment for high $V_{\max }$ halos. The number of subhalos in a host is typically smaller than the range of environments plotted, which is expected given that the virial radii of these halos are smaller than $2 h^{-1}$ Mpc. While checking for the effects of this bias, we found a direct correlation between the number of subhalos in a host and the local environment excluding the hosts own subhalos which may drive the trend between $R_{-}$and the local halo environment. This is consistent with the results of Wechsler et al. (2006).

\section{DISCUSSION}

This section discusses potential physical mechanisms behind the results presented in the previous section and compares the MS to theoretical and observational results from the literature. Our results highlight that tidal stripping, dynamical friction, and sub-sub interactions all impact the major merger rate.

\subsection{Groups and Clusters}

Tidal stripping can impact both the sub-sub and host-sub $R_{\mathrm{mm}}$. Tidal stripping in the MS, as measured using $\log \left(n_{\mathrm{bp}} / n_{\mathrm{bp}}\left(V_{\max }\right)\right)$ versus $r_{o} / r_{v}$ (see Section 3.2 and Figures 2 and 3) is consistent with theoretical expectations. First, the efficiency of tidal stripping is independent of $V_{s} / V_{h}$, with the exception of $V_{s} / V_{h} \approx 1$. Assuming the host halo and subhalo share a self-similar density profile, e.g., a Navarro-Frenk-White 

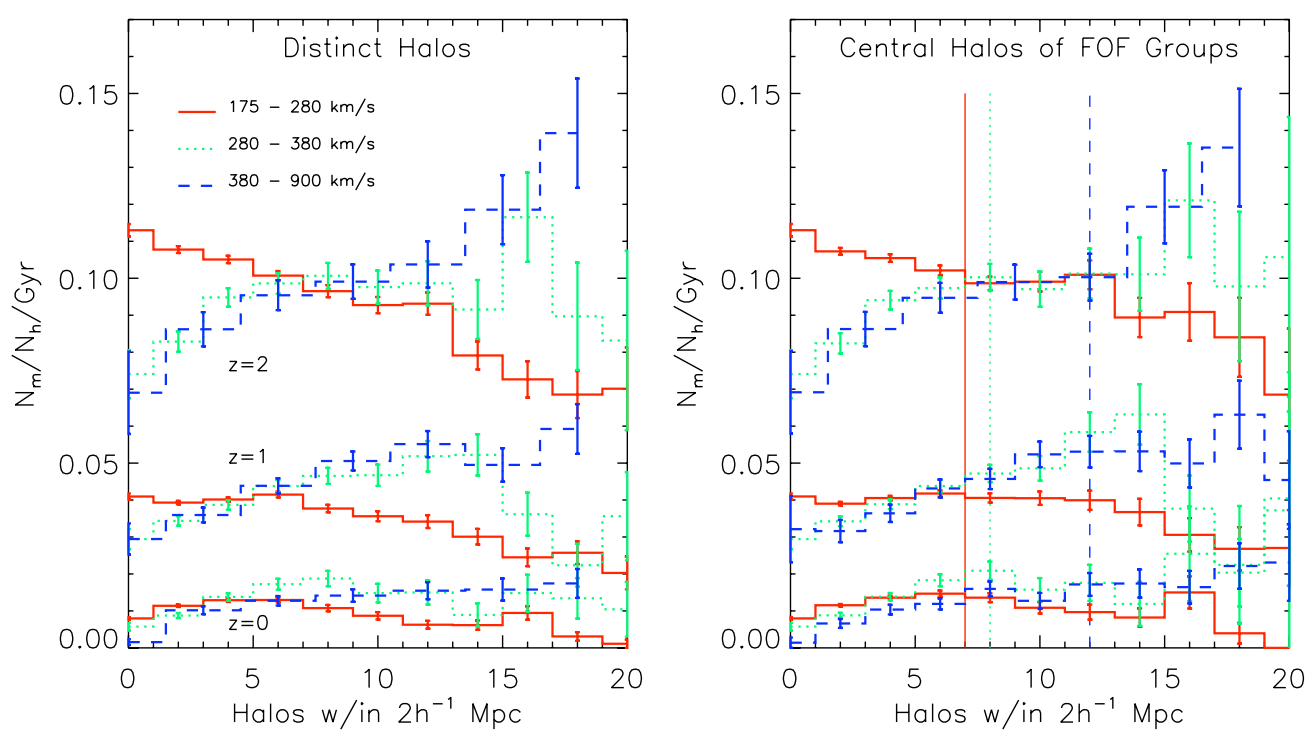

Figure 8. Major merger rate $R_{-}$vs. local halo environment for halos grouped by $V_{\max }$. In both panels, $R_{-}$is plotted vs. environment at $z=0,1$, and 2 (from bottom to top) for halos in three $V_{\max }$ ranges, as indicated. The left panel includes all distinct halos regardless of FOF group status. The right panel includes only halos that are the central halo of their FOF group. Vertical lines mark the environment below which $\approx 80 \%$ of the central halos reside.

(A color version of this figure is available in the online journal.)

(NFW) profile, a simple analytical model of tidal stripping based on the assumption of circular orbits predicts that $m_{\mathrm{ts}} / m_{\mathrm{vs}}=$ $M_{h}\left(r_{o}\right) / M_{h}\left(r_{v}\right)$, where $M_{h}(r)$ denotes the mass of the host halo within $r$. Note the efficiency of tidal stripping (defined by $m_{\mathrm{ts}} / m_{\mathrm{vs}}$ or $\left.n_{\mathrm{bp}} / n_{\mathrm{bp}}\left(V_{\mathrm{max}}\right)\right)$ is independent of mass in this simplest model. A refined analytical estimate that adopts a noncircular subhalo orbit and somewhat mass-dependent halo density profiles, both of which are determined using $N$-body simulations, is presented in Equation (8) of Mamon (2000). The diagonal lines in the center and right panels of Figure 2 correspond to this analytical estimate in groups and clusters, respectively, once $m_{\mathrm{ts}} / m_{\mathrm{vs}}$ is set equal to $n_{\mathrm{bp}} / n_{\mathrm{bp}}\left(V_{\max }\right)$. The analytical model is surprisingly consistent with the MS.

Tidal stripping is a cyclical process in which the stripping of the outer halo, tidal shocking at pericentric passages, and shocking during encounters with other subhalos trigger the relaxation of the inner halo. This ongoing relaxation allows for continual tidal stripping. The analytical estimates do not take this process into account, but using the subhalos' current $V_{s}$, as opposed to the value at accretion, appears to compensate for this.

The shapes of the contours in Figures 2 and 3 are also consistent with a tidal stripping interpretation. The broad scatter about the linear relationship between $\log \left(n_{\mathrm{bp}} / n_{\mathrm{bp}}\left(V_{\max }\right)\right)$ and $r_{o} / r_{v}$ is reflective of variations in $r_{o} / r_{\text {peri }}$, orbit cirularity, and dark matter halo concentrations. The general asymmetry of the contours reflects a predominance of subhalos that have had a pericentric passage over subhalos on their first infall. The bulge toward low $\log \left(n_{\mathrm{bp}} / n_{\mathrm{bp}}\left(V_{\max }\right)\right)$ and high $r_{o} / r_{v}$ likely represents a combination of subhalos on radial orbits that have passed through pericenter and subhalos that were previously the subhalos of lower $V_{\max }$ hosts. That is, they have experienced gravitational preprocessing.

We measure a low sub-sub $R_{\mathrm{mm}}$ in groups even though our subhalos have relatively large $V_{s}$. If the cross section for sub-sub mergers was dependent on the subhalos' current $V_{s}$, the analytical models presented in Section 1.1 would predict that the sub-sub $R_{\mathrm{mm}}$ for these subhalos should be enhanced. While Kravtsov et al. (2004b) found that subhalos lie on the same $m-V_{\max }$ relation as distinct halos, we find subhalos have suppressed $n_{\mathrm{pb}}$ compared to distinct halos. The suppressed sub-sub $R_{\mathrm{mm}}$ measured in the MS suggests that the cross section for major mergers correlates not with $V_{s}$ or with the mass within the tidal radius but with the actual bound mass of the subhalo.

One can check observationally that major mergers are dominated by host-sub mergers and that the sub-sub $R_{\mathrm{mm}}$ is suppressed. McIntosh et al. (2008) identify morphologically disturbed close pairs of galaxies in groups. They found that a slight majority of major mergers involve the central galaxy of a group and that the specific major merger rate for central galaxies is 3-4 times that for satellite galaxies. This is consistent with our results. They also found that the sub-sub specific merger rate declines with the mass of the host group. Their groups have an average of $\approx 5$ members and all have multiple subhalos.

Our results using the MS are consistent with previous studies of the interplay between tidal stripping and dynamical friction. Tidal stripping occurs on a shorter timescale than dynamical friction and is very effective at suppressing dynamical friction. Boylan-Kolchin et al. (2008) consider this problem by running high-resolution simulations of a lone subhalo that eventually merges with its host. They find that the merger timescale for major mergers ranges from $\approx 3.5$ to $7 \mathrm{Gyr}$, with longer timescales for lower $m_{\mathrm{vs}} / M_{\mathrm{vh}}$ and higher circularities. Major mergers are dominated by mergers with initial mass ratios closer to $3: 1$ than 1:1 indicating that typical major mergers involving a lone subhalo occur on extremely long timescales. Our results are roughly consistent with this, indicating the MS captures this interplay. Assuming that all host halos with a single subhalo at $z=1$ whose single subhalo has $V_{s} / V_{h}>0.7$ experience a merger at $z=0$ correctly predicts the major merger rate among halos with a single subhalo at $z=0$. This is clearly a very rough consistency check.

We find that introducing a second subhalo increases the host-sub $R_{+}$by a factor of 10 . This is an indication that the presence of a second subhalo can alter the orbit of the LMP subhalo, decreasing its orbital decay time. Under the assumptions of linear theory, subhalo orbits do not correlate with either the number of subhalos in a host at the time of accretion or 
the probability of accreting further subhalos. Halos do not know how lumpy the dark matter distribution is. If the subhalos remain independent objects, then doubling the number of subhalos should simply double the number of host-sub mergers. That $R_{+}$in fact increases dramatically suggests that subhalos interact with each other.

While mergers between subhalos are rare, unbound collisions are likely quite common. If these collisions were elastic, subhalos orbiting in similar directions could effectively trade angular momentum, but the angular momenta of subhalos traveling in opposite directions could not effectively cancel. An analytical treatment of subhalo orbits that assumed inelastic collisions found that these collisions do not enhance the host-sub merger rate (Peñarrubia \& Benson 2005). Sub-sub interactions between dynamic subhalos are, however, extremely elastic, as indicated by the mass lost during these interactions (Knebe et al. 2006). In this case, subhalos with opposing angular momenta can effectively dump both orbital energy and angular momentum with respect to the host halo during a sub-sub collision. Accordingly, the presence of one or several other subhalos of comparable mass or $V_{s}$ may significantly shorten the host-sub merger timescale. This scenario requires further study, but is physically plausible and consistent with our results.

We characterize subhalos by their current $V_{s}$ rather than by their mass or $V_{\max }$ at the time of accretion. This has advantages, particularly when studying tidal stripping and sub-sub mergers. It has the disadvantage, however, that some subhalos with $V_{s} / V_{h}>0.7$ at the time of accretion drop below this limit before the host-sub merger occurs, and we miss these major mergers. Sub-sub interactions promote mass loss, and we therefore expect to miss a greater proportion of the "true" major mergers in hosts with $n_{s} \geqslant 2$. The enhancement of $R_{\mathrm{mm}}$ in these halos therefore appears robust, but we may be missing more subtle effects. This particular analysis should therefore be redone using $V_{\max }$ at the time of accretion, which is likely a better indicator of subhalo luminosity than the current $V_{s}$ (Conroy et al. 2006).

Previous theoretical results confirm the excess of companions on small scales. Thacker et al. (2006) study the AGN-galaxy cross correlation in a smoothed particle hydrodynamics (SPH) simulation in which AGNs are fueled by major mergers. They find an excess at small scales compared to the galaxy-galaxy correlation. In another $N$-body simulation, Wetzel et al. (2009) also find an enhancement in the small-scale clustering of remnants of recent major mergers and attribute this enhancement to host-sub major mergers occurring preferentially in hosts with multiple subhalos. (Wetzel et al. 2009 was published after this paper was initially submitted.) They used their own $\mathrm{N}$-body simulation and defined major mergers using preaccretion masses, thus indicating that this result is robust to simulation issues. It should be noted that they find this excess lasts only $500 \mathrm{Myr}$ after the merger. It is unclear whether the merger of the second subhalo with the host is physical or the result of artificial disruption (see, for example, Kitzbichler \& White 2008). The companion subhalos are more prone to artificial disruption than the LMP subhalo and the physical excess clustering may persist for longer than 500 Myr. This question should be addressed with a higher resolution simulation.

On the observational side, Goto (2005) cross-correlated 266 E+A galaxies in the Sloan Digital Sky Survey (SDSS) with the SDSS imaging catalog and found that $\mathrm{E}+\mathrm{A}$ galaxies have an excess of companions on scales $<150 \mathrm{kpc}$ when compared to normal galaxies. Serber et al. (2006) perform a similar analysis for AGNs and find an excess of companions on similar scales. (See, however, the discussion in Padmanabhan et al. 2008. Note that the companions we predict are the less luminous subhalos of the major merger remnant and are unlikely to be the luminous red galaxies analyzed in the main text of Padmanabhan et al. 2008.) Previous studies of companion frequencies have suggested that tidal interactions with the companion trigger activity. We offer an alternative scenario in which activity is triggered by a major merger which is facilitated by the observed companion. The companion galaxies must also have lost considerable angular momentum which is consistent with small separations from the major merger remnant.

\subsection{Major Merger Rate and the Assembly Bias}

We measure no independent correlation between $R_{-}$and environment or $V_{\max }$, but do observed a correlation with local environment at fixed $V_{\max }$. The sense and size of this correlation is similar to so-called assembly biases.

It may be coincidental that $R_{\mathrm{mm}}$ does not correlate with environment or $V_{\max }$, and a sample with a lower $V_{\max }$ cutoff may show a stronger dependence on environment. A weak correlation between the major merger rate and the halo mass has been measured previously in the MS (Fakhouri \& Ma 2008). The absence of this trend in the results presented here may be due to the scatter between halo mass and $V_{\max }$.

Deviations from linear theory, which predicts formation history is independent of environment at fixed halo mass, have been measured in simulations for properties such as halo formation time, concentration, number of subhalos, subhalo mass function, and halo angular momentum (Gao et al. 2005; Wechsler et al. 2006; Croton et al. 2007; Gao \& White 2007; Wetzel et al. 2007). The correlation between $R_{-}$and environment at fixed $V_{\max }$ may be another facet of these socalled assembly biases; these correlations are all related to the halos' assembly histories. Previous studies have all found that the size of the assembly bias is small, and the results here are consistent with this.

Fakhouri \& Ma (2009) observe an increase in the FOF "halo" merger rate with environment at all halo masses, seemingly in conflict with our results. We are measuring the merger rate of subhalos, rather than FOF groups, and have adopted an environment measure which is both more local $\left(2 h^{-1} \mathrm{Mpc}\right.$ versus $7 \mathrm{Mpc}$ ) and considers halo counts rather than dark matter overdensities. We might term the FOF group merger rate an accretion rate, which we expect to correlate with $R_{-}$. It does not necessarily measure accretion onto the virialized central halos, however. In high-density environments, halos can be spuriously linked into FOF groups. At $z=0, R_{-}$for galaxylike central halos of FOF groups declines with environment in high-density environments, but increases somewhat in typical environments (see vertical lines in Figure 8). On 7 Mpc scales, the overdensity is less sensitive to the highest overdensities and may be dominated by this positive correlation. A measure which is sensitive to the highest overdensities may, however, be a better indicator of the bias.

Linear theory is based on the spherical collapse model, in which uniform shells of dark matter evolve radially under the influence of gravity. Tidal forces and the orbits and interactions of previously virialized halos within these shells are neglected. The role of tidal forces has been previously acknowledged; the halo mass function can be predicted correctly only if tidal forces are included in an average sense (Sheth \& Tormen 1999). These 
are analogs of the dynamics within host halos which likely contribute to the nonlinear trends seen in larger simulations.

Tidal stripping within FOF groups is occurring beyond the virial radii of the central halos. This is seen in the MS (Figures 2 and 3) and is predicted by the analytical estimate of Mamon (2000). Weak tidal forces on the outskirts of central halos likely suppress accretion onto and thereby the major merger rate of non-central halos. The correlation between $R_{-}$and environment for galaxy-like halos is significantly weakened when only central halos of FOF groups, which are less likely to be tidally influenced by a more massive halo, are considered. When the average $R_{-}$in the top $20 \%$ of environments, weighted by the environment distribution of central halos to correct for the trend with environment, is computed, $R_{-}$for distinct galaxylike halos is lower than for central halos at the $3 \sigma$ level at all three redshifts. An insignificant, $1 \sigma$, decrease is seen for group-like halos at $z=0$ and 1 .

Halos which are destined to be accreted by a more massive halo have always been bound to the future host halo; their orbital energy with respect to the host halo has always been negative. These halos are not on radial orbits. Major mergers in simulations have a range of initial orbital parameters, with a distribution of orbital circularities, $j / j_{c}(E)$, that peaks near 0.5 (Khochfar \& Burkert 2006). Accordingly, nonlinear effects such as dynamical friction and unbound collisions between future subhalos, both of which are likely enhanced in highdensity regions, may cause these bound halos to be prematurely accreted when compared to linear theory. It follows that central halos in dense environments will have enhanced accretion rates, subhalo counts, and major merger rates. This assumes the central halo is not within the tidal region of a more massive halo. In a large $N$-body simulation, Wetzel et al. (2007) measure an enhanced clustering for massive (group-like and above) halos that have recently accreted a potential LMP, in agreement with this interpretation.

The key to understanding the assembly biases may be identifying the halos that dominate the dynamics of their local neighborhood. Nonlinear effects in dense environments accelerate halo assembly for dominant halos and suppress it for non-dominant halos. This is consistent with the evolution of the assembly biases. At any redshift, a large fraction of the halos above the downturn in the halo mass function will be dominant in all environments. Lower mass halos may be dominant in low-density regions, but are unlikely to dominate high-density regions. As structure evolves and the distribution of halo masses shifts upward, the mass at which this behavior switches between these regimes increases. This shifting is typical of the evolution of the assembly biases.

The major merger rate may provide a way to directly probe the assembly biases. Confronting the assembly biases with observations is challenging due to both the size of the effect and the difficulty in correlating the relevant halo properties with observables. One exception, counting subhalos/satellite galaxies, is complicated by a correlation with group mass estimators (Berlind et al. 2006). A deeper understanding of the dynamics of subhalos may also contribute to a physical understanding of observed assembly-type biases. For example, Berlind et al. (2006) find that groups whose central galaxies are bluer than average, or rather less red than average, are more strongly clustered on large scales than average groups. Bluer galaxies have younger stellar ages, but it is unclear how this correlates with dark matter halo properties. We find that dense environments correlate with higher subhalo counts and by extension a decrease in the average time between subhalo accretion and a host-sub merger. These mergers may be more likely to bring young stars, and possibly gas, to the central galaxy.

\subsection{Completeness of the Subhalo Sample}

In order to determine when our results may be impacted by the artificial disruption of subhalos, we apply the criteria of Klypin et al. (1999), which were discussed in Section 2.1, to the relevant subhalos. We begin by determining whether our results are limited by mass resolution or force/spatial resolution. For the MS, a tidal radius of two times the spatial resolution corresponds to $10 h^{-1} \mathrm{kpc}$. Assuming, for simplicity, a static subhalo density profile subhalos in the MS drop below the 30 particle limit before their tidal radius reaches $10 \mathrm{~h}^{-1} \mathrm{kpc}$. A typical halo with $V_{\max }=100 \mathrm{~km} \mathrm{~s}^{-1}$, the lowest relevant $V_{\max }$, that has been stripped to the 30 particle limit has a tidal radius significantly larger than $10 h^{-1} \mathrm{kpc}$, so this conclusion likely holds in the realistic case that stripping alters the subhalo density profile. To check this conclusion we follow subhalos in the bound particle number versus orbital radius plane and find the redshift at which subhalos with $V_{\max }=120 \mathrm{~km} \mathrm{~s}^{-1}$, the lower limit of our catalog, begin dropping below the 30 particle limit. We do not appear to be losing any subhalos before this point. For each of the minimum $V_{\max }$ values considered below, the same result applies; the limiting condition is that a typical subhalo must retain at least 30 bound particles in order to avoid artificial disruption.

To estimate if and when subhalos with a given $V_{\max }$ drop below the 30 particle limit we use the analytical estimate of Mamon (2000) for tidal stripping of NFW halos,

$$
m_{t s} / m_{v s} \approx a_{r}\left(r_{o} / r_{\mathrm{vh}}\right)^{b_{r}}
$$

where $a_{r}$ and $b_{r}$ were chosen to reflect departures of the halo density profile from self-similarity. As can be seen in Figure 2, this provides a fair description of tidal stripping in the MS. By using the correlation measured in the MS between $V_{\max }$ and bound particle number for distinct halos, we can estimate the $r_{0} / r_{\mathrm{vh}}$ to which a typical halo with a given $V_{\max }$ retains 30 particles. This relation evolves with redshift, and subhalos of a given $V_{\max }$ are lost at increasing $r_{0} / r_{\mathrm{vh}}$ as the redshift increases. The pertinent $V_{\max }$ are $100 \mathrm{~km} \mathrm{~s}^{-1}$ for sub-sub major mergers with remnant $V_{\max }>175 \mathrm{~km} \mathrm{~s}^{-1}, 120 \mathrm{~km} \mathrm{~s}^{-1}$ for subhalo counts, and $210 \mathrm{~km} \mathrm{~s}^{-1}$ for host-sub major mergers in "grouplike" halos with $V_{\max } \geqslant 380 \mathrm{~km} \mathrm{~s}^{-1}$. In the simulation, $90 \%$ of the LMPs of major mergers have $V_{\max }$ values greater than 0.6 times that of the merger remnant. Hence, we use values of $100 \mathrm{~km} \mathrm{~s}^{-1}$ and $210 \mathrm{~km} \mathrm{~s}^{-1}$ to check for the completion of sub-sub and host-sub merger remnants. Typical subhalos with $V_{\max }=210 \mathrm{~km} \mathrm{~s}^{-1}$ survive numerical effects to $r_{o} / r_{\mathrm{vh}}=0.1$ out to $z>4$. Subhalos with $V_{\max }=120 \mathrm{~km} \mathrm{~s}^{-1}$ survive to $r_{o} / r_{\mathrm{vh}}<0.1$ at $z=0, r_{o} / r_{\mathrm{vh}} \approx 0.2$ at $z=1$, and $r_{o} / r_{\mathrm{vh}} \approx 0.4$ at $z=2$. Typical subhalos with $V_{\max }=100 \mathrm{~km} \mathrm{~s}^{-1}$ survive to $r_{o} / r_{\mathrm{vh}} \approx 0.25$ at $z=0$ and $r_{o} / r_{\mathrm{vh}} \approx 0.5$ at $z=1$.

These estimates show that we are clearly pushing the ability to track subhalos in the MS. Most of the important results, however, are quite robust against the effects of subhalo completion. The largest exception is the case of major mergers between subhalos. Subhalo mergers are more likely to happen before subhalos are significantly tidally stripped, which mitigates the effects of losing potential least massive merger progenitors to artificial evaporation at moderate radii. That said, the result that subhalo 
major mergers are rare must rest solely on results from redshifts near $z=0$. The most robust result is that the presence of multiple subhalos drastically reduces the timescale for host-sub major mergers. We postulate that it is the presence of bound structures in the simulation that drives the major merger rate. It is therefore appropriate to separate halos based on the presence or absence of such a structure, regardless of whether a subhalo would exist in the hypothetical case of better mass resolution. The related result that an assembly-type bias is seen in $R_{-}$is somewhat less robust as it depends on identifying the particular halos in which subhalo counts and the host-sub $R_{-}$are enhanced. The fidelity of this identification starts fading near $z \approx 1$ as subhalos with $V_{\max } \approx 120 \mathrm{~km} \mathrm{~s}^{-1}$ begin to be subject to artificial evaporation. In the absence of a correlation between environment and the probability of a subhalo evaporating, however, this effect only introduces noise. This may degrade the measured trend but cannot create it. To the extent that environment and subhalo loss may be correlated, the initial bound particle counts of fresh subhalos are likely suppressed in overdense environments, biasing against the result seen.

The evolution of $R_{\mathrm{mm}}$ for halos with $V_{\max } \geqslant 175 \mathrm{~km} \mathrm{~s}^{-1}$ may be strongly affected by subhalo incompletion. Completion affects both the measured evolution of $R_{+}$and $R_{-}$in all environments and the comparison between environments. The first subhalos lost to numerical effects are not the LMP halos of the host-sub major mergers, which dominate $R_{\mathrm{mm}}$. Rather, they are the lower $V_{\max }$ subhalos which facilitate the host-sub major mergers. These halos are not missed by the halo finder; they are truly absent, having been artificially dissolved. This results in an $R_{\mathrm{mm}}$ that is increasingly underestimated at high redshifts; an effect that becomes important for galaxy-like halos at lower redshifts than group-like halos. In galaxy-like halos, a second effect comes into play when the LMPs of host-sub major mergers are artificially dissolved, resulting in a major merger occurring prematurely. The combination of these effects may be quite complex, yet in Figure $1, R_{\mathrm{mm}}$ for all halos shows a smooth evolution. Subhalos also play a role in group identification. As completion begins to affect subhalos with $V_{\max } \approx 120 \mathrm{~km} \mathrm{~s}^{-1}$, the requirement that a "group" halo must have at least two subhalos becomes increasingly stringent. As some "real groups" are not identified, the physical density of major mergers in groups is underestimated at high redshift and the measured slope of the evolution is too shallow. The flattening of the physical number density of major mergers in groups seen in Figure 1 is likely in part due to completion. The average number of subhalos in the groups also declines with redshift, which may be real, artificial, or a combination. Note that these effects refer only to the evolution $R_{\mathrm{mm}}$ in groups. As long as the sub-sub $R_{-}$ is significantly lower than the host-sub $R_{-}, R_{-}$in groups will be suppressed compared to all halos.

\section{CONCLUSIONS}

We have made a detailed study of the environments of major mergers in the MS (Springel et al. 2005c). Our goal in doing so is to provide a theoretical background for observational tests both to confront the dark matter simulations directly and to identify populations of major merger remnants through their environments. We now summarize our results and predictions for the environments of major mergers. Comparing simulation results with observations requires some caution. The most robust observational tests rely on physically motivated comparisons between actual observations. Such tests are likely to be insensitive to the details of the simulation, and we focus on results of this type.
The main results are as follows: the specific backward looking $R_{\mathrm{mm}}$ is suppressed for sub-sub mergers compared to distinct halos selected above the same $V_{\max }$. As subhalos dominate numerically in groups, the specific $R_{\mathrm{mm}}$ among group members is also suppressed compared to isolated halos. This is despite the fact that the specific host-sub $R_{\mathrm{mm}}$ is enhanced in groups. Merger remnants are significantly more likely than the average halo to host a subhalo. The observational consequence of this is that the frequency of faint nearby companions should be enhanced for major merger remnants. Similarly, when crosscorrelated with a fainter galaxy sample, major merger remnants should show an enhanced correlation on scales less than the virial radius of the remnants. The specific major merger rate was found to be uncorrelated with both local environment and $V_{\max }$ when each was studied independently. There is no physical motivation for this result. At fixed $V_{\max }$, however, we found an assembly-type bias in the backward-looking specific major merger rate. For galaxy-like halos, $R_{-}$decreases with increasing density while for group-like halos it increases. A potential physical driver for the major merger assembly bias was advanced in Section 4. Based on this result, we predict that major merger remnants that are the central galaxies of groups should show enhanced clustering on scales beyond the viral radius of the group. Major mergers are a promising way of observing an assembly-type bias in as much as major mergers can be identified observationally.

The physically motivated predictions presented above can be used to confront the simulation results with observations. As major mergers constitute a small fraction of all halos, major mergers should be conservatively identified to ensure that observed "major mergers" are dominated by true major mergers while the non-merger population will always be dominated by non-mergers. Using environment to test whether other populations are major merger remnants will be complicated by the physics of these objects. Three populations of interest are starbursts, $\mathrm{K}+\mathrm{A}$ galaxies, and AGNs. The correlation between dark matter major mergers and these populations depends on the presence of substantial amounts of gas during the merger, which we do not attempt to track in the simulation.

Given the complicating gas physics, studying the frequency of fainter companions has clear advantages over clustering studies. When studying small scales, i.e., the one-halo term, crosscorrelations weigh the contribution of each central galaxy by the number of subhalos, $n_{s}$. If the likelihood of being able to fuel a starburst or an AGN correlates with $n_{s}$, then observing an enhanced clustering will depend on the sign and strength of this correlation. When studying larger scales, the two-halo term, observing the predicted enhancement for group-like halos requires that gassy major mergers are a fair sample of all major mergers, which is likely not the case. In contrast, studying the frequency of fainter companions does not require a fair sampling and weights each central halo equally.

Designing specific observational tests based on the results presented here is a topic for future work.

This work was partially funded by NSF grant 0707731 and NASA ATP grant NNX08AH30G. The authors thank E. Scannapieco for useful discussions and G. A. Mamon for useful comments on an earlier version of this paper. J.A.H. thanks S. Malhaotra, J. Rhoads, and the Arizona State University School of Earth and Space Exploration for their hospitality while this work was being completed. 
The Millennium Simulation databases used in this paper and the Web application providing online access to them were constructed as part of the activities of the German Astrophysical Virtual Observatory.

The particular structure of the database design which allows efficient querying for merger trees is described in Lemson \& Springel (2006).

\section{REFERENCES}

Alexander, D. M., Bauer, F. E., Chapman, S. C., Smail, I., Blain, A. W., Brandt, W. N., \& Ivison, R. J. 2005, ApJ, 632, 736

Barnes, J. E. 2004, MNRAS, 350, 798

Barnes, J. E., \& Hernquist, L. 1992, ARA\&A, 30, 705

Berlind, A. A., Kazin, E., Blanton, M. R., Pueblas, S., Scoccimarro, R., \& Hogg, D. W. 2006, ApJS, 167, 1

Best, P. N., Kaiser, C. R., Heckman, T. M., \& Kauffmann, G. 2006, MNRAS, 368, L67

Blanton, M. R., Berlind, A. A., \& Hogg, D. W. 2007, ApJ, 664, 791

Bond, J. R., Cole, S., Efstathiou, G., \& Kaiser, N. 1991, ApJ, 379, 440

Borys, C., Smail, I., Chapman, S. C., Blain, A. W., Alexander, D. M., \& Ivison, R. J. 2005, ApJ, 635, 853

Bower, R. G., Benson, A. J., Malbon, R., Helly, J. C., Frenk, C. S., Baugh, C. M., Cole, S., \& Lacey, C. G. 2006, MNRAS, 370, 645

Boylan-Kolchin, M., Ma, C.-P., \& Quataert, E. 2008, MNRAS, 383, 93

Collister, A. A., \& Lahav, O. 2005, MNRAS, 361, 415

Conroy, C., Wechsler, R. H., \& Kravtsov, A. V. 2006, ApJ, 647, 201

Cox, T. J., Jonsson, P., Primack, J. R., \& Somerville, R. 2006, MNRAS, 373 1013

Croton, D. J., Gao, L., \& White, S. D. M. 2007, MNRAS, 374, 1303

Croton, D. J., et al. 2006, MNRAS, 365, 11

De Lucia, G., Kauffmann, F., Springel, V., White, S. D. M., Lanzoni, B., Stoehr, F., Tormen, G., \& Yoshida, N. 2004, MNRAS, 348, 333

Di Matteo, T., Springel, V., \& Hernquist, L. 2005, Nature, 433, 604

Fabian, A. C., Sanders, J. S., Taylor, G. B., Allen, S. W., Crawford, C. S., Johnstone, R. M., \& Iwasawa, K. 2006, MNRAS, 366, 417

Fakhouri, O., \& Ma, C.-P. 2008, MNRAS, 386, 577

Fakhouri, O., \& Ma, C.-P. 2009, MNRAS, 394, 1825

Ferrarese, L., \& Merritt, D. 2000, ApJ, 539, L9

Gao, L., Springel, V., \& White, S. D. M. 2005, MNRAS, 363, L66

Gao, L., \& White, S. D. M. 2007, MNRAS, 377, 5

Gebhardt, K., et al. 2000, ApJ, 539, L13

Gerssen, J., van der Marel, R. P., Axon, D., Mihos, J. C., Hernquist, L., \& Barnes, J. E. 2004, AJ, 127, 75

Ghinga, S., Moore, B., Governato, F., Lake, G., Quinn, T., \& Stadel, J. 1998, MNRAS, 300, 146

Goto, T. 2005, MNRAS, 357, 937

Gottlöber, S., Klypin, A., \& Kravtsov, A. V. 2001, ApJ, 546, 223

Hayashi, E., Navarro, J. F., Taylor, J. E., Stadel, J., \& Quinn, T. 2003, ApJ, 584, 541

Hopkins, P. F., Hernquist, L., Cox, T. J., Di Matteo, T., Robertson, B., \& Springel, V. 2006, ApJS, 163, 1

Jogee, S. 2005, in AGN Physics on All Scales, ed. D. Alloin, R. Johnson, \& P. Lira (Lecture Notes in Physics; Berlin: Springer), chap. 6

Kauffmann, G., \& Haehnelt, M. 2000, MNRAS, 311, 576

Kauffmann, G., \& White, S. D. M. 1993, MNRAS, 261, 921

Khochfar, S., \& Burkert, A. 2006, A\&A, 445, 403

Kitzbichler, M. G., \& White, S. D. M. 2008, MNRAS, 391, 1489

Klimentowski, J. L., Łokas, E. L., Kazantzidis, S., Mayer, L., \& Mamon, G. A. 2009, MNRAS, 397, 2015

Klypin, A., Gottlober, S., Kravtsov, A. V., \& Khokhlov, A. M. 1999, ApJ, 516, 530

Knebe, A., Power, C., Stuart, P. D. G., \& Gibson, B. K. 2006, MNRAS, 368 , 741

Komossa, S., Burwitz, V., Hasinger, G., Predehl, P., Kaastra, J. S., \& Ikebe, Y. 2003, ApJ, 582, L15
Kravtsov, A. V., Berlind, A. A., Wechsler, R. H., Klypin, A. A., Gottlöber, S., Allgood, B., \& Primack, J. R. 2004a, ApJ, 609, 35

Kravtsov, A. V., Gnedin, O. Y., \& Klypin, A. A. 2004b, ApJ, 609, 482

Lacey, C., \& Cole, S. 1993, MNRAS, 262, 627

Lemson, G., \& Springel, V. 2006, in ASP Conf. Ser. 351, Astronomical Data Analysis Software and Systems XV, ed. C. Gabriel, C. Arviset, D. Ponz, \& E. Solano (San Francisco, CA: ASP), 212

Maccio, A. V., Moore, B., Stadel, J., \& Potter, D. 2006, in Proc. XLIst Rencontres de Moriond, XXVIth Astrophysics Moriond Meeting: From Dark Halos to Light, ed. L. Tresse, S. Maurogordato, \& J. Tran Thanh Van (Gif-sur-Yvette: Editions Frontieres), in press

Magorrian, J., et al. 1998, AJ, 115, 2285

Makino, J., \& Hut, P. 1997, ApJ, 481, 83

Mamon, G. A. 1992, ApJ, 401, L3

Mamon, G. A. 2000, in ASP Conf. Ser. 197, Dynamics of Galaxies: from the Early Universe to the Present, ed. F. Combes, G. A. Mamon, \& V. Charmandaris (San Francisco, CA: ASP), 377

Mandelbaum, R., Seljak, U., Kauffmann, G., Hirata, C., \& Brinkman, J. 2006, MNRAS, 368, 715

Marconi, A., \& Hunt, L. K. 2003, ApJ, 589, L21

McIntosh, D. H., Guo, Y., Hertzberg, J., Katz, N., Mo, H. J., van den Bosch, F C., \& Yang, X. 2008, MNRAS, 388, 1537

McLure, R. J., \& Dunlop, J. S. 2002, MNRAS, 331, 795

Mihos, J. C., \& Hernquist, L. 1996, ApJ, 464, 641

Naab, T., \& Burkert, A. 2003, ApJ, 597, 893

Natarajan, P., De Lucia, G., \& Springel, V. 2007, MNRAS, 376, 180

Norton, S. A., Gebhardt, K., Zabludoff, A. I., \& Zaritsky, D. 2001, ApJ, 557, 150

Owers, M., Blake, C., Couch, W., Pracy, M. B., \& Bekki, K. 2007, MNRAS, 381,494

Padmanabhan, N., White, M., Norberg, P., \& Porciani, C. 2008, MNRAS, 397, 1862

Peñarrubia, J., \& Benson, A. J. 2005, MNRAS, 364, 977

Press, W., \& Schechter, P. 1974, ApJ, 187, 425

Sanders, D. B., \& Mirabel, I. F. 1996, ARA\&A, 34, 749

Sargent, A. I., Sanders, D. B., \& Phillips, T. G. 1989, ApJ, 346, L9

Sargent, A. I., et al. 1987, ApJ, 312, L35

Scoville, N. Z., et al. 1986, ApJ, 311, L47

Serber, W., Bahcall, N., Menard, B., \& Richards, G. 2006, ApJ, 643, 68

Sheth, R. K., \& Tormen, G. 1999, MNRAS, 308, 119

Somerville, R., \& Kolatt, T. 1999, MNRAS, 305, 1

Spergel, D. N., et al. 2003, ApJS, 148, 175

Springel, V. 2000, MNRAS, 312, 859

Springel, V., Di Matteo, T., \& Hernquist, L. 2005a, ApJ, 620, L79

Springel, V., Di Matteo, T., \& Hernquist, L. 2005b, MNRAS, 361, 776

Springel, V., White, S. D. M., Tormen, G., \& Kauffmann, G. 2001, MNRAS, 328,726

Springel, V., et al. 2005c, Nature, 435, 629

Steinmetz, M., \& Navarro, J. F. 2002, New Astron., 7, 155

Tasitsiomi, A., Kravtsov, A. V., Wechsler, R. H., \& Primack, J. R. 2004, ApJ, 614,533

Tasitsiomi, A., Wechsler, R. H., Kravtsov, A. V., \& Klypin, A. A. 2008, ApJ, submitted

Thacker, R. J., Scannapieco, E., \& Couchman, H. M. P. 2006, AJ, 653, 86

Toomre, A., \& Toomre, J. 1972, ApJ, 178, 623

Tremaine, S., et al. 2002, ApJ, 574, 740

Wechsler, R. H., Zentner, A. R., Bullock, J. S., Kravtsov, A. V., \& Allgood, B. 2006, ApJ, 652, 71

Wetzel, A. R., Cohn, J. D., \& White, M. 2009, MNRAS, 394, 2182

Wetzel, A. R., Cohn, J. D., White, M., Holz, D. E., \& Warren, M. S. 2007, ApJ, 656,139

White, S. D. M. 1996, in Cosmology and Large-Scale Structure, ed. R. Schaeffer, J. Silk, \& J. Zinn-Justin (Dordrecht: Elsevier Science), p. 349

Wyithe, J. S. B., \& Loeb, A. 2002, ApJ, 581, 886

Wyithe, J. S. B., \& Loeb, A. 2003, ApJ, 595, 614

Yang, Y., Zabludoff, A. I., Zaritsky, D., Lauer, T. R., \& Mihos, J. C. 2004, ApJ, 607, 258

Yoachim, P., \& Dalcanton, J. J. 2008, ApJ, 682, 1004 